\section{Observation: Case Report}

\section{Inflammatory Response Cells During Acute Respiratory Distress Syndrome in Patients With Coronavirus Disease 2019 (COVID-19)}

Background: A previous report of lung biopsies from a patient with coronavirus disease 2019 (COVID-19) and acute respiratory distress syndrome (ARDS) identified mononuclear cell infiltration but not the type of mononuclear cells (1).

Objective: To describe the type of immune cells identified by imaging mass cytometry in lung tissue from 2 patients with COVID-19 and fatal ARDS.

Case Reports: The first patient was a 94-year-old woman who had close contact with a confirmed case of COVID-19 eleven days earlier. She presented with an 8-day history of lethargy and fever (maximum temperature, $39{ }^{\circ} \mathrm{C}$ ), and her history included 10 years of coronary heart disease. Physical examination found wheezing in both lungs and peripheral edema, and chest radiography identified right pulmonary infiltration and consolidation with right pleural effusion. She had a C-reactive protein level of $115.1 \mathrm{mg} / \mathrm{L}$ (normal level, <3 $\mathrm{mg} / \mathrm{L}$ ) and leukocyte count of $10.2 \times 10^{9}$ cells/L with $9 \%$ lymphocytes. Tests processed by the Beijing Centers for Disease Control confirmed that she had COVID-19. At the direction of her relatives, we provided noninvasive therapy that included supplemental oxygen and symptomatic treatment. She gradually developed dyspnea and hypoxemia and had a fatal cardiac arrest on day 10 of the illness.

The second patient was a 65-year-old man whose wife had COVID-19. He presented with a 4-day history of dry cough, anorexia, and fever (maximum temperature, $38.6^{\circ} \mathrm{C}$ ). On admission, computed tomography of the chest showed bilateral pneumonia. He had a C-reactive protein level of 13.5 $\mathrm{mg} / \mathrm{L}$ and a leukocyte count of $3.0 \times 10^{9}$ cells/L with $27.4 \%$ lymphocytes. Tests processed by the Beijing Centers for Disease Control confirmed that he had COVID-19. We initiated supportive therapy and administered moxifloxacin to prevent secondary infection. On day 15 of the illness, he required invasive ventilatory support. We changed his antibiotics to vancomycin and imipenem for suspected sepsis and administered intravenous methylprednisolone and immune globulin to attenuate systemic inflammation. On day 16 , he had a C-reactive protein level of $244.4 \mathrm{mg} / \mathrm{L}$, leukocyte count of $10.1 \times 10^{9}$ cells/L with $1.6 \%$ lymphocytes, and blood lactic acid level of $3.13 \mathrm{mmol} / \mathrm{L}$. He developed septic shock and died on day 21 of the illness.

Lung tissue was obtained by autopsy in both patients and by percutaneous biopsy in a control patient, a 54-year-old man with a pulmonary nodule. When we examined tissues stained with hematoxylin-eosin, the first patient had the typical histologic features of ARDS, with diffuse alveolar damage, alveolar septum edema, epithelial cell proliferation, hyaline membrane formation, infiltration of lymphocytes and monocytes into interstitial and alveolar spaces, and a small number of neutrophils. The second patient had the typical histologic features of bacterial pneumonia, with alveolar damage; alveolar septum edema; epithelial cell proliferation; and desquamation of pneumocytes, cellular fibromyxoid exudates, many phagocytes, neutrophil debris, and pus cells in alveolar cavities. The control patient had infiltration of interstitial inflammatory cells.

Imaging mass cytometry on lung tissue from the first patient (Figure) found diffuse infiltration of CD4 T lymphocytes, macrophages (CD68), and a focal infiltration of natural killer cells (CD16 and CD107A). Tissue from the second patient had a cluster infiltration of neutrophils (CD11b and CD16) and activated macrophages (Arg1), a diffuse infiltration of mature $T$ cells (CD45RA and CD4), and a scattered infiltration of natural killer cells and dendritic cells (CD276 and CD14), which distributed differently from macrophages and were accompanied by local overexpression of type 1 collagen. The distributions of infiltration by macrophages, mature $T$ cells, and natural killer cells in the 2 patients were different, as was the expression of type 1 collagen, and the second patient had more dendritic cells. Both patients had relatively independent distributions of cell subsets by t-distributed stochastic neighbor embedding and PhenoGraph analysis. The first patient had more infiltration by immune cells. This patient had a cluster distribution of mature T cells (CD45RA) and macrophages. The second patient had a cluster distribution of mature $T$ cells (CD45RA and CD45RO) and macrophages, which correlated with bacterial infection. More CD45RA ${ }^{+} \mathrm{T}$ cells were recruited in the first patient, whereas mostly $C D 45 \mathrm{RO}^{+} \mathrm{T}$ cells were recruited in the second patient. Additional data and figures are available from the authors on request.

Discussion: Two previous studies of lung tissue from patients with severe acute respiratory syndrome found mononuclear cell infiltration and hyaline membrane formation $(2,3)$, whereas 1 previous study of a patient with COVID-19 and ARDS found interstitial infiltration by mononuclear cells (1). Our study suggests that these infiltrated mononuclear cells are CD4 T cells, CD8 T cells, natural killer cells, and macrophages; that recruitment of aberrant CD45RA ${ }^{+} T$ cells is the immunologic feature of COVID-19; and that once bacterial pneumonia occurs, some phagocytes recruited by CD4 T cells begin to play a major role in lung injury. We conclude that additional studies are needed to understand the immune response in patients with COVID-19.

Yulin Zhang, $P h D, M D^{*}$

Yuxue Gao, MD*

Luxin Qiao, MD*

Wenjing Wang, PhD

Dexi Chen, PhD, MD

Beijing Institute of Hepatology, Beijing You An Hospital, Capital Medical University

Beijing, China

* Drs. Zhang, Gao, and Qiao contributed equally to this work.

This article was published at Annals.org on 13 April 2020. 


\section{LETTERS}

Figure. Imaging mass cytometry with markers of interest.
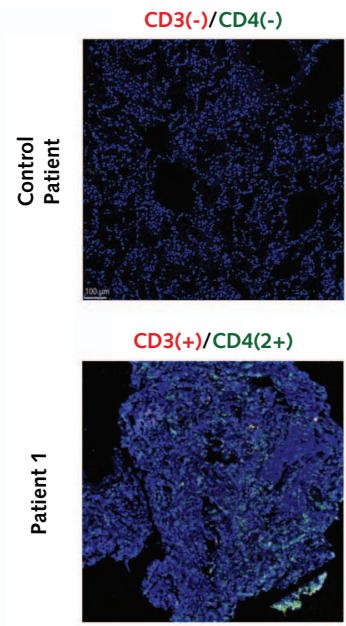

$\mathrm{CD} 3(+) / \mathrm{CD} 4(+)$

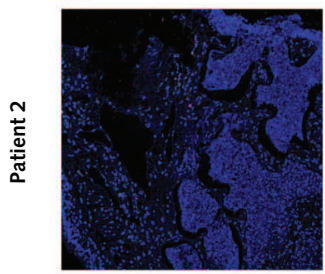

CD68(+)

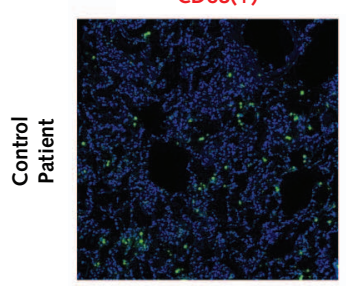

CD68(4+)

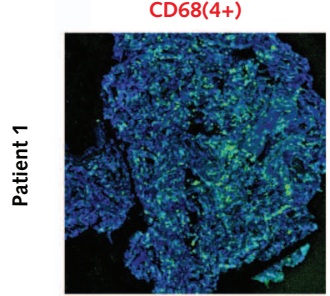

CD68(4+)

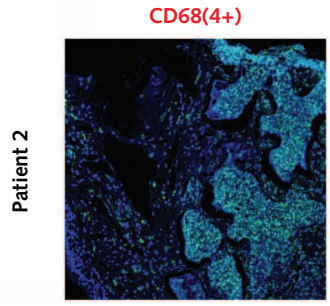

$\mathrm{CD} 3(-) / \mathrm{CD} 8(+)$

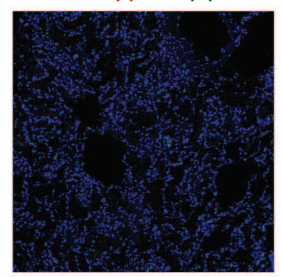

$\mathrm{CD} 3(+) / C D 8(+)$

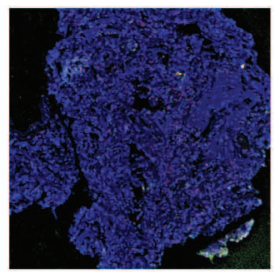

$\mathrm{CD} 3(+) / \mathrm{CD} 8(+)$

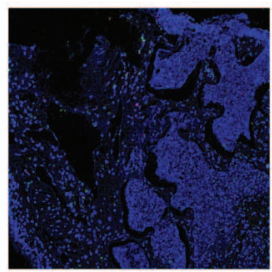

CD20(+)/B-catenin(-)

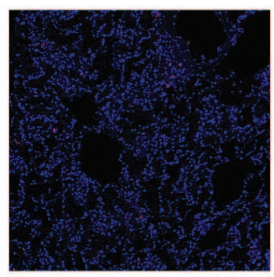

CD20(+)/B-catenin(+)

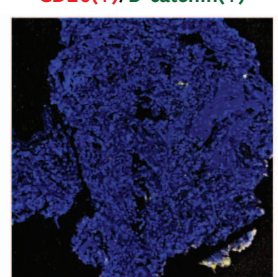

CD20(+)/B-catenin(-)



CD45RA(+)/CD4(-)

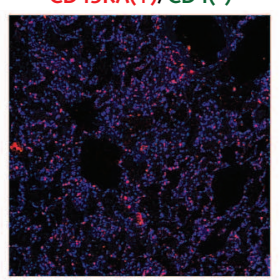

CD45RA(3+)/CD4(2+)

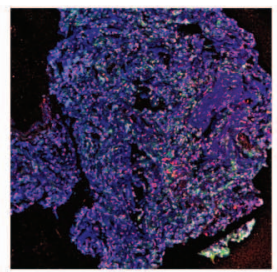

CD45RA(4+)/CD4(+)

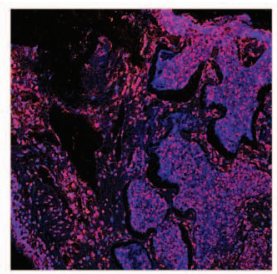

CD25(-)/CD4(-)/FOXP3(-)

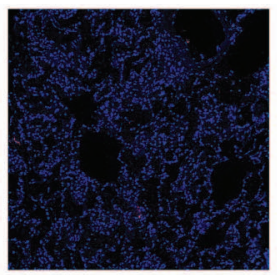

CD25(+)/CD4(2+)/FOXP3(+)

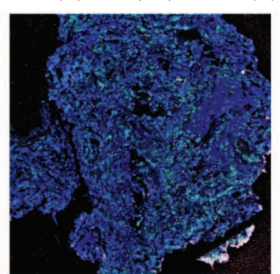

$\mathrm{CD} 25(+) / C D 4(+) / F O X P 3(-)$



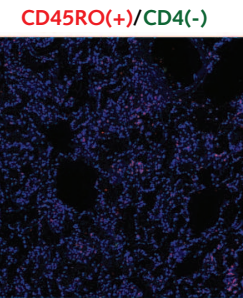

$\mathrm{CD} 45 \mathrm{RO}(+) / \mathrm{CD} 4(2+)$



$\mathrm{CD} 45 \mathrm{RO}(+) / \mathrm{CD} 4(+)$

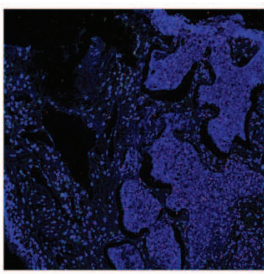

CD276(-)

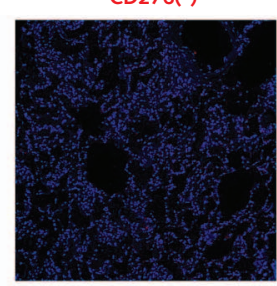

CD276(+)

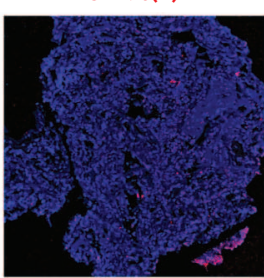

CD276(2+)

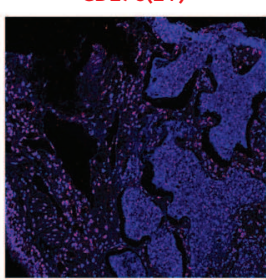

CD16(-)/CD107A(+)

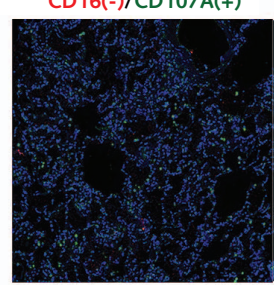

$\mathrm{CD} 16(2+) / \mathrm{CD} 107 \mathrm{~A}(2+)$

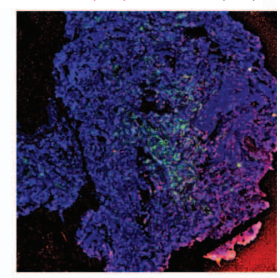

$\mathrm{CD} 16(+) / \mathrm{CD} 107 \mathrm{~A}(2+)$

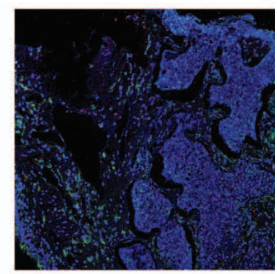

Collagen1(-)/E-cadherin(-)



Collagen1(3+)/E-cadherin(+)

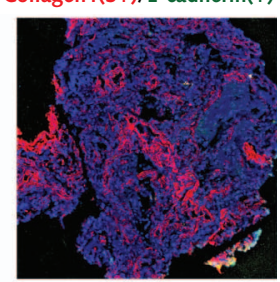

Collagen1(2+)/E-cadherin(+)

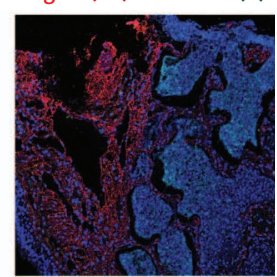

Representative mass cytometry images for each panel are different colors, and expression levels are in parentheses. Iridium-DNA staining is shown in blue. All images are from the same tissue sections. We designed a metal isotope-labeled antibody panel to detect multiple markers simultaneously in 1 slide by using the imaging mass cytometry system. For detailed methods, please refer to the Imaging Mass Cytometry Staining Protocol for FFPE Sections on the Fluidigm website (http://cn.fluidigm.com/search?query=IMC+Staining+Protocol\&resourceTypes=protocol). All raw data were acquired using a Hyperion Imaging System (Fluidigm). The resulting files were in MCD format and were exported as TIFF files using the MCD Viewer. FFPE = formalin-fixed paraffin-embedded. 
Note: This study was done in accordance with regulations issued by the National Health Commission of China and was approved by the local Commission of Ethics (reference number StV 12-2005).

Grant Support: By the National Natural Science Foundation of China (grants 81873761 and 81672026) and National Science and Technology Major Special Program of the 13th Five-Year Plan of China (grants 2018ZX10302104 and 2018ZX10302205).

Disclosures: Authors have disclosed no conflicts of interest. Forms can be viewed at www.acponline.org/authors/icmje/ConflictOflnterest Forms.do?msNum=L20-0227.
Corresponding Author: Dexi Chen, PhD, MD, Beijing Institute of Hepatology, Beijing You An Hospital, Capital Medical University, Beijing 100069, China; e-mail, dexichen@ccmu.edu.cn.

doi:10.7326/L20-0227

\section{References}

1. Xu Z, Shi L, Wang Y, et al. Pathological findings of COVID-19 associated with acute respiratory distress syndrome. Lancet Respir Med. 2020;8:420-422. [PMID: 32085846] doi:10.1016/S2213-2600(20)30076-X

2. Nicholls JM, Poon LL, Lee KC, et al. Lung pathology of fatal severe acute respiratory syndrome. Lancet. 2003;361:1773-8. [PMID: 12781536]

3. Ding $Y$, Wang $H$, Shen $H$, et al. The clinical pathology of severe acute respiratory syndrome (SARS): a report from China. J Pathol. 2003;200:282-9. [PMID: 12845623] 